\title{
Estado nutricional e desenvolvimento da linguagem em crianças de uma creche pública
}

\author{
Nutritional status and language development in children from \\ a public day care center
}

\author{
Juliana Nunes Santos', Stela Maris Aguiar Lemos², Joel Alves Lamounier ${ }^{3}$
}

\begin{abstract}
RESUMO
Objetivo: Investigar a associação entre estado nutricional e desenvolvimento da linguagem infantil. Métodos: Participaram do estudo 34 crianças de quatro a seis anos e 11 meses de idade de creches de Belo Horizonte, Minas Gerais. As crianças foram avaliadas quanto ao desenvolvimento da linguagem receptiva e expressiva utilizando-se as tarefas do instrumento de Avaliação de Desenvolvimento de Linguagem (ADL). Para a avaliação nutricional foram utilizados os índices estatura/idade e peso/idade, de acordo com os padrões e curva de crescimento da OMS. Resultados: Em relação à linguagem, 13 (38\%) apresentaram alterações; e quanto ao estado nutricional, 26 crianças (76\%) eram eutróficas, seis (18\%) estavam em risco nutricional, uma (3\%) estava desnutrida e uma (3\%) tinha sobrepeso. Ao comparar as médias dos percentis estatura/idade e peso/idade entre as crianças com e sem distúrbio do desenvolvimento da linguagem, não se encontrou diferença. Conclusão: Não foi observada associação entre estado nutricional e desenvolvimento da linguagem nas crianças. Contudo, os percentis estatura/idade e peso/idade foram, tendenciosamente, menores no grupo de crianças com distúrbio de linguagem.
\end{abstract}

Descritores: Estado nutricional; Linguagem; Criança; Desenvolvimento infantil; Desenvolvimento da linguagem

\section{INTRODUÇÃO}

O desenvolvimento neuropsicomotor da criança resulta da interação entre características biológicas e fatores socioculturais. Assim, pode ser influenciado, direta ou indiretamente, pelas inúmeras variantes biológicas ou socioculturais mais presentes em um ou outro momento dessa interação ${ }^{(1)}$.

Nos países em desenvolvimento, a desnutrição crônica (redução na velocidade de crescimento com peso e altura deficitários para a idade) é considerada o maior fator de risco para alterações no desenvolvimento infantil, juntamente com a estimulação cognitiva inadequada, deficiência de iodo e anemia ferropriva $^{(2)}$.

De uma perspectiva global, a extensão das deficiências nu-

Trabalho realizado no Núcleo de Pós-graduação da Faculdade de Medicina da Universidade Federal de Minas Gerais - UFMG - Minas Gerais (MG), Brasil. (1) Doutora, Professora Adjunto do Curso de Fonoaudiologia da Universidade Federal de Minas Gerais - UFMG - Minas Gerais (MG), Brasil.

(2) Doutora, Professora Adjunto do Curso de Fonoaudiologia da Universidade Federal de Minas Gerais - UFMG - Minas Gerais (MG), Brasil.

(3) Doutor, Professor titular do Departamento de Pediatria da Faculdade de Medicina da Universidade Federal de Minas Gerais - UFMG - Minas Gerais (MG), Brasil.

Endereço para correspondência: Juliana Nunes Santos. R. Coronel Pedro Jorge, 170/201, Prado, Belo Horizonte, Minas Gerais (MG), Brasil. CEP: 30410-350. E-mail: jununessantos@yahoo.com.br

Recebido em: 18/11/2009; Aceito em: 1/4/2010 tricionais e a perda do potencial de desenvolvimento cognitivo são indicadas por números alarmantes. Em crianças abaixo de cinco anos, estima-se que 112 milhões estão abaixo do peso, 178 milhões tem desnutrição crônica e mais de 219 milhões não alcançaram seu potencial de desenvolvimento, o que repercute em prejuízos para essas crianças, para suas famílias e para as sociedades das quais participam ${ }^{(3)}$.

Das deficiências nutricionais, a desnutrição energéticoproteica (caracterizada pelo pobre status nutricional), tem sido considerada um problema de saúde até mesmo nos países mais desenvolvidos ${ }^{(4,5)}$. Ela está relacionada à inadequada dieta energética e sua ocorrência na população infantil repercute negativamente no rendimento escolar e no desenvolvimento cognitivo dessa população, podendo se prolongar até a vida $\operatorname{adulta}^{(6,7)}$.

Os efeitos negativos da desnutrição podem ser entendidos ao se considerar o desenvolvimento encefálico, pois o cérebro humano desenvolve-se rapidamente nos primeiros anos de vida. Aumenta de tamanho com a idade e atinge a forma adulta entre os seis e os 14 anos. Nesse período de rápido crescimento, existem maiores demandas de nutrientes necessários ao desenvolvimento cerebral. Se a nutrição é inadequada, a estrutura do cérebro pode ficar comprometida ${ }^{(6,8)}$.

Durante o desenvolvimento infantil habilidades particulares são rapidamente adquiridas ${ }^{(9)}$. Se a ocorrência da desnutrição inviabiliza o funcionamento metabólico optimal durante 
um período mais sensível do desenvolvimento da linguagem, então podem existir consequências negativas permanentes ${ }^{(6)}$.

As alterações de linguagem afetam 5\% a 10\% de todas as crianças $^{(10)}$ e estão relacionadas ao baixo desempenho escolar e à exclusão social. Tais alterações podem ser causadas por fatores sociais, ambientais e biológicos ${ }^{(10-12)}$. Desnutrição, por exemplo, é um fator de etiologia biológica, mas que pode resultar de um entrelaçamento de fatores sociais e ambientais ${ }^{(3)}$.

Além da desnutrição, dentre os fatores de etiologia biológica, destacam-se as perdas auditivas e as alterações neurológicas ${ }^{(6,13-15)}$.

A relação das deficiências nutricionais com a linguagem ainda é pouco descrita na literatura nacional. Portanto, este estudo vem ao encontro dessa necessidade, já que se propõe a investigar uma associação entre estado nutricional e desenvolvimento da linguagem infantil.

\section{MÉTODOS}

Pesquisa aprovada pelo Comitê de Ética em Pesquisa da Faculdade de Medicina da Universidade Federal de Minas Gerais (UFMG), sob protocolo 380/05.

Trata-se de um estudo transversal descritivo parte de uma coorte de crianças monitoradas pelo Projeto Saúde de Ferro em uma creche pública de Belo Horizonte. O projeto, de caráter social e de relevância no âmbito da saúde coletiva, é desenvolvido pelo Núcleo de Pós-graduação da Faculdade de Medicina da UFMG e atua na prevenção da anemia ferropriva em crianças matriculadas nas creches de Belo Horizonte.

A creche em estudo é responsável pelo cuidado em tempo integral de crianças de baixo nível socioeconômico, cuja matrícula na instituição é dependente da comprovação de trabalho e renda dos pais. A instituição, para realizar suas atividades, recebe auxílio financeiro da Prefeitura e da Igreja Católica local. As crianças realizam suas principais refeições (café da manhã, almoço e jantar) na creche.

Fizeram parte da amostra 34 crianças entre quatro e seis anos e 11 meses de idade (média 65,5 \pm 9,5 meses) que não apresentavam alterações cognitivas, impedimentos motores de fala, distúrbios emocionais significativos e sintomatologia neurológica; não possuíam história de prematuridade, anóxia, infecção ou outras intercorrências perinatais e apresentavam avaliação auditiva adequada. A creche possui atualmente 139 crianças atendidas em tempo integral, no entanto, somente as 34 crianças da amostra estavam sendo monitoradas pelo projeto saúde de ferro, e, portanto, fizeram parte da presente análise ${ }^{(14)}$.

Dos participantes, $20(59 \%)$ pertencem ao gênero masculino e 14 (41\%), ao feminino.

\section{Procedimentos}

Os pais das crianças receberam esclarecimentos sobre $o$ caráter voluntário do estudo, seus objetivos e repercussões e assinaram o Termo de Consentimento Livre e Esclarecido. No mesmo momento, responderam a um questionário contendo dados de identificação e informações acerca da história de vida e do desenvolvimento da criança.

As medidas antropométricas (peso e estatura) foram co- letadas por estagiários do curso medicina, de acordo com as técnicas estabelecidas pela Organização Mundial da Saúde ${ }^{(16)}$, sob supervisão de um médico pesquisador do estudo. Para obter o peso corporal, as crianças se apresentavam com o mínimo de roupas. $\mathrm{O}$ peso foi verificado em balança digital eletrônica, com capacidade para $150 \mathrm{~kg}$ e divisão de $100 \mathrm{~g}$. As crianças foram medidas em pé, descalças com estadiômetro.

Para avaliar o estado nutricional (por meio do método antropométrico), utilizou-se a curva de crescimento da $O M S^{(17)}$, mais atualizada e apropriada para o acompanhamento do crescimento $^{(18)}$. Os índices antropométricos utilizados para avaliar o estado nutricional foram: estatura para idade (E/I) e peso para idade $(\mathrm{P} / \mathrm{I})$, medidos em percentis e escores $\mathrm{Z}$. Adotou-se como ponto de corte valor $<-2$ escores $\mathrm{Z}$ para desnutrição (baixo peso e baixa estatura/idade), valores entre $-2 \mathrm{e}$ -1 escores para a indicação de risco nutricional e $\geq+2$ escores $\mathrm{Z}$ para sobrepeso ${ }^{(16,19)}$.

As crianças foram avaliadas quanto ao desenvolvimento da linguagem por uma única fonoaudióloga, utilizando-se as tarefas do instrumento de Avaliação de Desenvolvimento de Linguagem (ADL), proposto para identificar alterações na aquisição e desenvolvimento da linguagem ${ }^{(20)}$. A avaliação foi realizada em uma sala da creche, própria para a observação, em sessão individual de aproximadamente 20 minutos. Os registros das respostas referentes aos comportamentos esperados para cada idade foram feitos em fichas individuais.

A ADL é um instrumento clínico, padronizado e validado, prático e eficiente para avaliar o conteúdo e a estrutura da linguagem na faixa etária de um ano a seis anos e 11 meses. A administração da ADL é individual e avalia os domínios receptivos e expressivos da linguagem. O conteúdo da linguagem é verificado por meio de tarefas concentradas nos conceitos de quantidade, qualidade (adjetivos), relação espacial, temporal e sequência. A estrutura da linguagem é avaliada por meio de tarefas referentes à morfologia e à sintaxe ${ }^{(20)}$.

\section{Análise de dados}

Para a entrada, o processamento e a análise quantitativa dos dados, foi utilizado o software SPSS - versão 14.0. Para a análise antropométrica, foram utilizados os softwares WHOAnthro e WhoAnthroPlus, versão $3^{(17,21)}$. Para fins de análise descritiva, foi feita distribuição de frequência das variáveis categóricas envolvidas na avaliação da linguagem em estudo e análise das medidas de tendência central e de dispersão das variáveis contínuas. Na análise estatística, empregaram-se os testes Qui-quadrado e Exato de Fisher para verificar diferenças entre as proporções e o teste t de Student para comparar variáveis contínuas entre os participantes distribuídos em dois grupos, de acordo com a presença do distúrbio de linguagem.

\section{RESULTADOS}

Em relação à linguagem, 38\% dos participantes apresentaram alterações nos resultados da avaliação indicativas de um distúrbio na aquisição e desenvolvimento da linguagem.

Os resultados da avaliação antropométrica mostraram que $76 \%$ das crianças são eutróficas, $18 \%$ estão em situação 
Tabela 1. Características das crianças apresentadas em dois grupos segundo a presença do distúrbio da linguagem

\begin{tabular}{|c|c|c|c|c|c|c|c|}
\hline \multirow{3}{*}{ Características } & \multicolumn{4}{|c|}{ Desenvolvimento de linguagem } & \multirow{3}{*}{$\begin{array}{l}\text { Média da } \\
\text { diferença }\end{array}$} & \multirow{3}{*}{ Teste $\mathrm{t}$} & \multirow{3}{*}{ Valor de $\mathrm{p}$} \\
\hline & \multicolumn{2}{|c|}{ Normal $(\mathrm{N}=21)$} & \multicolumn{2}{|c|}{ Distúrbio $(\mathrm{N}=13)$} & & & \\
\hline & Média & $\mathrm{DP}$ & Média & DP & & & \\
\hline Idade das crianças (meses) & 67,8 & $\pm 9,9$ & 63,2 & $\pm 8,3$ & 4,6 & 1,39 & 0,17 \\
\hline Percentil peso/idade & 54,2 & $\pm 21,2$ & 46,5 & $\pm 19,7$ & 7,4 & 1,04 & 0,30 \\
\hline Percentil altura/idade & 43,5 & $\pm 28,7$ & 35,7 & $\pm 27,2$ & 7,7 & 0,77 & 0,44 \\
\hline
\end{tabular}

Teste t de Student

Legenda: DP = desvio-padrão

Tabela 2. Estado nutricional e presença do distúrbio da linguagem

\begin{tabular}{|c|c|c|c|c|c|c|c|}
\hline \multirow{2}{*}{\multicolumn{2}{|c|}{ Características das crianças }} & \multicolumn{2}{|c|}{ Desenvolvimento de linguagem } & \multirow[b]{2}{*}{ Odds ratio } & \multirow[b]{2}{*}{ IC (95\%) } & \multirow{2}{*}{$\begin{array}{c}\text { Teste Exato de } \\
\text { Fisher }\end{array}$} & \multirow{2}{*}{$\begin{array}{l}\text { Valor } \\
\text { de } p\end{array}$} \\
\hline & & $\begin{array}{l}\text { Normal } \\
(\mathrm{N}=21)\end{array}$ & Distúrbio $(\mathrm{N}=13)$ & & & & \\
\hline \multirow{2}{*}{ Gênero } & Masculino & 10 & 10 & \multirow{2}{*}{0,27} & \multirow{2}{*}{$0,04-1,60$} & \multirow{2}{*}{2,76} & \multirow{2}{*}{0,15} \\
\hline & Feminino & 11 & 3 & & & & \\
\hline \multirow{3}{*}{$\begin{array}{l}\text { Estatura/idade } \\
\text { Estado nutricional }\end{array}$} & Eutrófico & 17 & 10 & \multirow{3}{*}{1,27} & \multirow{3}{*}{$0,18-9,04$} & \multirow{3}{*}{1,59} & \multirow{3}{*}{0,65} \\
\hline & Faixa de risco & 3 & 3 & & & & \\
\hline & Baixa estatura (desnutrido) & 1 & 0 & & & & \\
\hline \multirow{3}{*}{$\begin{array}{l}\text { Peso/ idade } \\
\text { Estado nutricional }\end{array}$} & Eutrófico & 18 & 13 & \multirow{3}{*}{0,00} & \multirow{3}{*}{$0,00-3,77$} & \multirow{3}{*}{2,03} & \multirow{3}{*}{0,36} \\
\hline & Faixa de risco & 2 & 0 & & & & \\
\hline & Sobrepeso & 1 & 0 & & & & \\
\hline
\end{tabular}

Teste Exato de Fisher

Legenda: $I C$ = intervalo de confiança

de risco nutricional, uma criança está desnutrida e outra tem sobrepeso, de acordo com os padrões da $\mathrm{OMS}^{(17,21)}$.

As medidas antropométricas em percentis, os valores da hemoglobina e a idade das crianças alocadas em dois grupos segundo a presença do distúrbio de linguagem podem ser verificadas na Tabela 1.

Os resultados das avaliações antropométricas utilizando-se escores $\mathrm{Z}$ para estatura/idade e peso/idade, anemia e gênero das crianças estão apresentados na Tabela 2. São distribuídos segundo a presença ou ausência do distúrbio de linguagem.

A Figura 1 permite a visualização das distribuições do índice antropométrico estatura/idade (E/I).

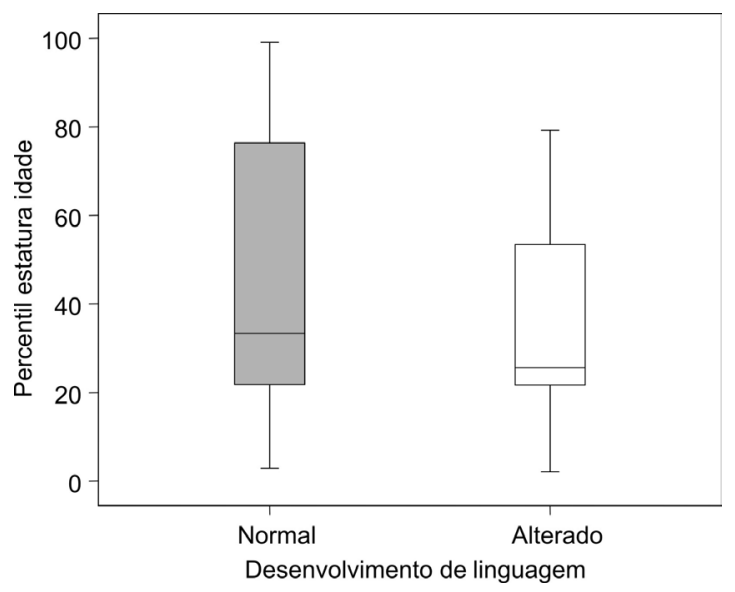

Figura 1. Distribuição do percentil estatura/idade (E/I) segundo o desenvolvimento da linguagem
A classificação dos distúrbios da linguagem (leve, moderado, severo) segundo o estado nutricional das crianças está representada na Figura 2.

\section{DISCUSSÃO}

O desenho de estudo transversal proporciona observar a situação nutricional e o desenvolvimento da linguagem no mesmo momento histórico. Por isso, não permite que uma relação causal direta seja estabelecida com os resultados encontrados. Somente uma associação entre as variáveis pode ser verificada. No entanto, a relevância e a magnitude da ocorrência dos distúrbios nutricionais ${ }^{(4)}$ e seu risco para o desenvolvimento infantil ${ }^{(2)}$ justificam esta investigação, especialmente na área da fonoaudiologia, carente desses estudos.

Os resultados da avaliação da linguagem mostraram que $38 \%$ das crianças avaliadas apresentam alteração na aquisição e desenvolvimento da linguagem, sendo que, destes, quatro apresentam um distúrbio severo. Outros estudos realizados em creches revelam resultados semelhantes ${ }^{(1,22)}$, o que aponta para a necessidade de uma integração maior entre os setores de educação e saúde, a fim de se criarem estratégias preventivas e promotoras de saúde nessas instituições, as quais são responsáveis pelo cuidado de grande parte das crianças brasileiras. Certamente, além da nutrição apropriada, a estimulação cognitiva e social adequadas são fundamentais para o futuro dessas crianças. Estudos longitudinais mostram que crianças que receberam estimulação cognitiva e social precoce têm melhor desempenho nas funções psicossociais, cognitivas 

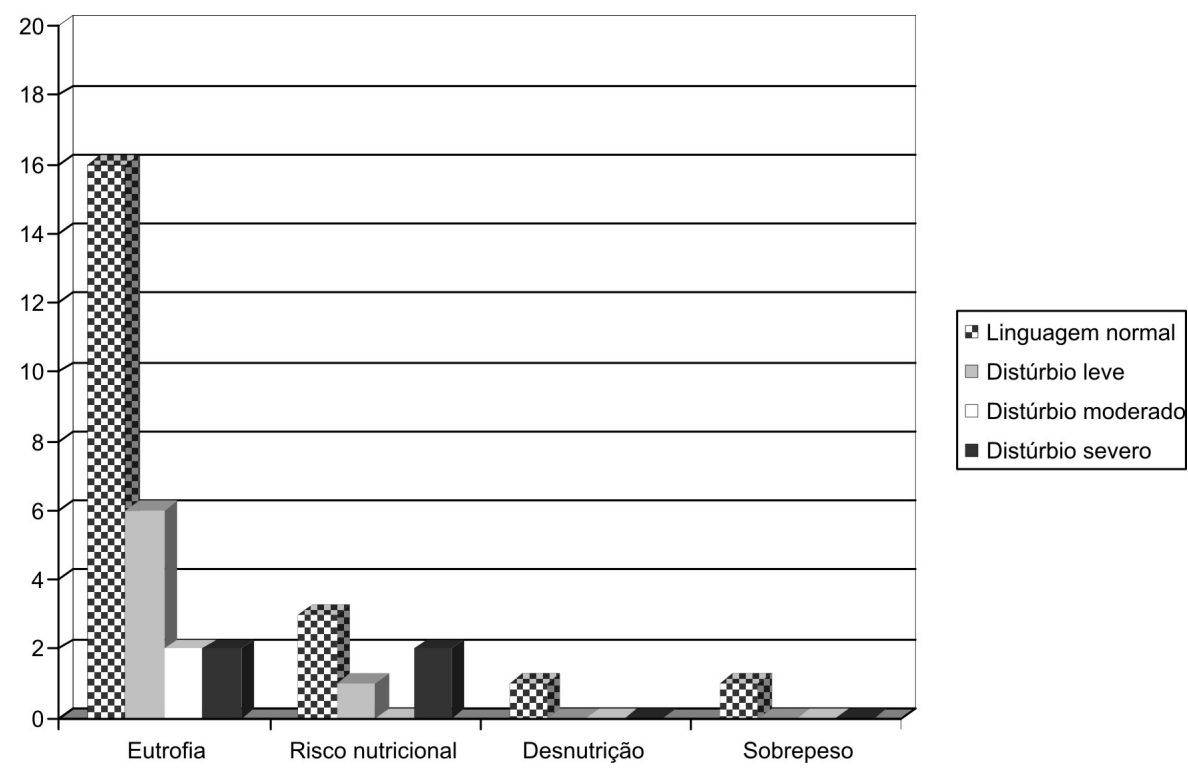

Figura 2. Classificação do grau de desenvolvimento da linguagem segundo o estado nutricional das crianças

e educacionais na adolescência do que aquelas desprovidas dessa estimulação( ${ }^{(3)}$.

Neste estudo, a maioria das crianças avaliadas apresenta bom estado nutricional (Tabela 2). Ou seja, são eutróficas e possuem peso e altura adequados para idade. A literatura recente mostra uma redução da prevalência da desnutrição nesta faixa etária decorrente de melhores condições socioeconômicas e de saneamento ${ }^{(23,24)}$. Deve-se considerar também o papel da instituição "creche", a qual oferece às crianças uma alimentação qualitativamente adequada, potencializando a diminuição dos riscos nutricionais.

No entanto, esse potencial institucional de promoção da saúde infantil das creches se depara com limitações biológicas, ambientais e sociais ${ }^{(2,13,25)}$ que podem ser considerados fatores de vulnerabilidade para alterações do desenvolvimento infantil. Das crianças avaliadas, $18 \%$ estão em risco nutricional e 3\% são desnutridas. Agrupando-se essas categorias, obtém-se um percentual de $21 \%$, o que equivale a aproximadamente $1 / 5$ da amostra. Quando se consideram os possíveis efeitos dessas condições no desenvolvimento e escolarização, cabe alertar para a necessidade de reduzir esses índices, aparentemente, ainda preocupantes. Pesquisa em creches da região leste de Belo Horizonte (MG) mostrou valores semelhantes: $18 \%$ de risco nutricional para estatura/ idade e $5 \%$ para desnutrição ${ }^{(26)}$.

Ao comparar as médias dos percentis estatura/idade e peso/ idade entre as crianças com e sem distúrbio do desenvolvimento da linguagem, não se encontrou diferença estatística (Tabela 1). No entanto, a observação da distribuição do índice E/I (Figura 1) retrata menores valores de percentis no grupo de crianças com linguagem alterada, o que apoia os achados da literatura ${ }^{(3)}$ e concorda com estudo realizado por fonoaudiólogas brasileiras, o qual verificou atraso no desenvolvimento fonológico de crianças com antecedentes de desnutriçãa ${ }^{(11)}$.

Além disso, os resultados visualizados na Figura 2 mostram para o grupo de crianças em situação de risco nutricional, uma distribuição diferente das categorias de linguagem relativa- mente àquela observada no subgrupo de criança eutróficas. No primeiro subgrupo, é mais alta a porcentagem de crianças com linguagem normal, mesmo quando se compara tais crianças com a porcentagem total do grupo com distúrbio (de leve a grave). No subgrupo em risco nutricional, não existe essa diferença, de modo que o número de crianças com linguagem normal é igual ao número de crianças com distúrbio. Assim, embora os testes estatísticos não tenham demonstrado diferenças significativas na direção esperada, os resultados descritivos obtidos merecem destaque, pois apontam para a relevância de se desenvolver novos estudos transversais, com amostras maiores e nas quais os subgrupos da nutrição venham a ser relativamente balanceados. Dessa maneira, possivelmente, poderá estabelecida uma relação entre as alterações da linguagem e as alterações nutricionais. Este conhecimento pode ser bastante significativo, especialmente para a questão da aprendizagem e desenvolvimento da linguagem, a fim de adotar práticas preventivas e promotoras de saúde nos contextos pré-escolar e escolar.

Estudos longitudinais de populações do Brasil, Guatemala, Índia, Filipinas e África do Sul mostraram que a desnutrição crônica entre os 12 e 36 meses de idade é importante preditor de desempenho cognitivo inadequado e/ou baixo aproveitamento escolar anual (séries cursadas e aprovadas) ${ }^{(7)}$. Ademais, a deficiência de proteínas e de micronutrientes peculiares ao quadro de desnutrição compromete o funcionamento normal do organismo, especialmente o das crianças, em fase de crescimento e amadurecimento neuronal, tornando-as mais vulneráveis aos efeitos maléficos da desnutrição no desenvolvimento ${ }^{(6,8)}$.

Este estudo pretendeu relacionar as medidas antropométricas ao desenvolvimento da linguagem. $\mathrm{O}$ fato de não ter sido encontrada uma associação com significância estatística na amostra estudada não implica, necessariamente, que as variáveis não tenham relação entre si. Explicação possível para esse resultado encontra-se no perfil nutricional das crianças avaliadas, em sua maioria eutróficas. São muito importantes, 
também, os estudos longitudinais voltados para o estudo da linguagem e da desnutrição, a fim de possibilitar o conhecimento do real impacto da carência nutricional no desenvolvimento da criança.

\section{CONCLUSÃO}

Os resultados mostraram que $38 \%$ das crianças apresentaram distúrbio na aquisição e desenvolvimento da linguagem. A maioria das crianças são eutróficas e $18 \%$ estão em situação de risco nutricional. A desnutrição e o sobrepeso foram observados em $3 \%$ da amostra.

Os valores dos percentis estatura/idade e peso/idade foram menores no grupo de crianças com distúrbio de linguagem. No entanto, essa diferença observada entre as medidas antroprométricas e o desenvolvimento da linguagem das crianças não teve significância estatística.

A caracterização da linguagem em diferentes subgrupos nutricionais sugere relações que merecem ser investigadas em outros estudos, com novas composições de amostras.

\begin{abstract}
Purpose: To investigate the association between nutritional status and child language development. Methods: The study included 34 children from four to six years and 11 months of age, enrolled in day care centers of Belo Horizonte, Minas Gerais, Brazil. Children were assessed for receptive and expressive language development using the tasks of the instrument Language Development Evaluation (ADL). Nutritional screening used the weight-for-age and length/height-for-age indexes, according to the WHO's Child Growth Standards. Results: Regarding language, 13 (38\%) children presented alterations; and, regarding nutritional status, 26 (76\%) were well-nourished, six (18\%) were at nutritional risk, one (3\%) was malnourished, and one (3\%) was overweight. When the means for the height/age and the weight/age indexes were compared between children with and without language development impairments, no difference was found. Conclusion: No association was observed between nutritional status and language development in the studied children. However, the indexes height-for-age and weight-for-age tended to be lower in children with language impairment.
\end{abstract}

Keywords: Nutritional status; Language; Child; Child development; Language development

\title{
REFERÊNCIAS
}

1. Biscegli TS, Polis LB, Santos LM, Vicentin M. Avaliação do estado nutricional e do desenvolvimento neuropsicomotor em crianças frequentadoras de creche. Rev Paul Pediatr. 2007;25(4):337-42.

2. Walker SP, Wachs TD, Gardner JM, Lozoff B, Wasserman GA, Pollitt E, Carter JA; International Child Development Steering Group. Child development: risk factors for adverse outcomes in developing countries. Lancet. 2007;369(9556):145-57. Comment in: Lancet. 2007;369(9563. Black MM, Walker SP, Wachs TD, Ulkuer N, Gardner JM, Grantham-McGregor S, Lozoff B, Engle PL, de Mello MC. Policies to reduce undernutrition include child development. Lancet. 2008;371(9611):454-5. Comment on: Lancet. 2008;371(9611):510-26.

4. Meier R, Stratton R. Basic concepts in nutrition: epidemiology of malnutrition. e-SPEN 2008;3(4):e167-70.

5. Heltberg R. Malnutrition, poverty, and economic growth. Health Econ. 2009;18 Suppl 1:S77-88.

6. Benton D; ILSI Europe a.i.s.b.l. The influence of children's diet on their cognition and behavior. Eur J Nutr. 2008;47 Suppl 3:25-37. Review.

7. Victora CG, Adair L, Fall C, Hallal PC, Martorell R, Richter L, Sachdev HS; Maternal and Child Undernutrition Study Group. Maternal and child undernutrition: consequences for adult health and human capital. Lancet. 2008;371(9609):340-57. Review. Erratum in: Lancet. 2008;371(9609):302.

8. Schweigert ID, Souza DOG, Perry MLS. Desnutrição, maturação do sistema nervoso central e doenças neuropsiquiátricas. Rev Nutr. 2009;22(2): 271-81

9. Ruben RJ. A time frame of critical/sensitive periods of language development. Acta Otolaryngol. 1997;117(2):202-5.

10. Vitto MMP, Feres MCLC. Distúrbios da comunicação oral em crianças. Medicina (Ribeirão Preto). 2005;38(3/4):229-34.

11. Lima LM, Queiroga BAM. Aquisição fonológica em crianças com antecedentes de desnutrição. Rev CEFAC. 2007;9(1):13-20.

12. Fattal-Valevski A, Azouri-Fattal I, Greenstein YJ, Guindy M, Blau A,
Zelnik N. Delayed language development due to infantile thiamine deficiency. Dev Med Child Neurol. 2009;51(8):629-34.

13. Maria-Mengel MRS, Linhares MBM. Risk factors for infant developmental problems. Rev Latinoam Enferm. 2007;15(Special):837-42.

14. Santos JN, Rates SPM, Lemos SMA, Lamounier JA. Anemia em crianças de uma creche pública e as repercussões sobre o desenvolvimento de linguagem. Rev Paul Pediatr. 2009;27(1):67-73.

15. Santos JN, Lemos SMA, Rates SPM, Lamounier JA. Habilidades auditivas e desenvolvimento de linguagem em crianças. Pró-Fono. 2008;20(4):255-60.

16. WHO Expert Committee on Physical Status: the Use and Interpretation of Anthropometry; World Health Organization. Physical status: the use of and interpretation of anthropometry, report of a WHO expert committee. Geneva; World Health Organization; 1995.

17. World Health Organization. Anthro for personal computers, version 3 , 2009: software for accessing growth and development of the world's children's. Geneva: World Health Organization; 2009.

18. Silveira FJF, Lamounier JA. Avaliação nutricional de crianças do Vale do Alto Jequitinhonha com a utilização das novas curvas de crescimento do NCHS e da OMS. Rev Paul Pediatr. 2009;27(2):133-8.

19. World Health Organization. WHO Child Growth Standards: methods and development. Length/height-for-age, weight-for-age, weight-for-length, weight-for-height and body mass index-for-age. Geneva: World Health Organization; 2006.

20. Menezes MLN. A construção de um instrumento para avaliação do desenvolvimento da linguagem: idealização, estudo piloto para padronização e validação [tese]. Rio de Janeiro: Instituto Fernandes Figueira; 2003.

21. World Health Organization. WHO AnthroPlus for personal computers manual: software for accessing growth of world's children's and adolescents. Geneva: World Health Organization; 2009.

22. Basílio CS, Puccini RF, Silva EMK, Pedromônico MRM. Living 
conditions and receptive vocabulary of children aged two to five years. Rev Saúde Pública = J Public Health. 2005;39(5):725-30.

23. Monteiro CA. A queda da desnutrição infantil no Brasil. Cad Saúde Pública = Rep Public Health. 2009;25(5):950.

24. Lamounier JA. Transição epidemiológica nutricional em crianças e adolescentes argentinos de áreas carentes. Rev Paul Pediatr. 2009;27(2):124-6.
25. Bradley RH, Corwyn RF. Socioeconomic status and child development. Annu Rev Psychol. 2002;53:371-99.

26. Rocha DS, Lamounier JA, Franceschini SCC, Capanema FD, Norton RC, Oliveira VB, et al. Fatores de risco para déficit nutricional em crianças matriculadas em creches. Rev Pediatr. 2007;8(1):17-26. 